\title{
Effects of Statin Plus Ezetimibe on Coronary Plaques in Acute Coronary Syndrome Patients with Diabetes Mellitus: Sub-Analysis of PRECISE-IVUS Trial
}

\author{
Koichiro Fujisue ${ }^{1}$, Kenshi Yamanaga1, Suguru Nagamatsu ${ }^{1}$, Hideki Shimomura², Takuro Yamashita ${ }^{3}$, \\ Koichi $\mathrm{Nakao}^{4}$, Sunao Nakamura ${ }^{5}$, Masaharu Ishihara ${ }^{6}$, Kunihiko Matsui ${ }^{7}$, Naritsugu Sakaino ${ }^{8}$,

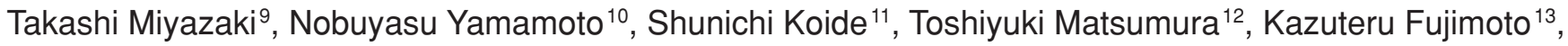 \\ Ryusuke Tsunoda ${ }^{14}$, Yasuhiro Morikami ${ }^{15}$, Koushi Matsuyama ${ }^{3}$, Shuichi Oshima ${ }^{16}$, Kenji Sakamoto ${ }^{1}$, \\ Yasuhiro Izumiya ${ }^{1,17}$, Koichi Kaikita ${ }^{1}$, Seiji Hokimoto ${ }^{1}$, Hisao Ogawa ${ }^{18}$ and Kenichi Tsujita ${ }^{1}$ \\ ${ }^{1}$ Department of Cardiovascular Medicine, Graduate School of Medical Sciences and Center for Metabolic Regulation of Healthy \\ Aging (CMHA), Kumamoto University, Kumamoto, Japan \\ ${ }^{2}$ Department of Cardiovascular Medicine, Fukuoka Tokushukai Medical Center, Kasuga, Japan \\ ${ }^{3}$ Division of Cardiology, Social Insurance Omuta Tenryo Hospital, Omuta, Japan \\ ${ }^{4}$ Division of Cardiology, Saiseikai Kumamoto Hospital Cardiovascular Center, Kumamoto, Japan \\ ${ }^{5}$ Interventional Cardiology Unit, New Tokyo Hospital, Matsudo, Japan \\ ${ }^{6}$ Department of Cardiovascular and Renal Medicine, Hyogo College of Medicine, Nishinomiya, Japan \\ ${ }^{7}$ Department of Community Medicine, Kumamoto University, Kumamoto, Japan \\ ${ }^{8}$ Division of Cardiology, Amakusa Medical Center, Amakusa, Japan \\ ${ }^{9}$ Division of Cardiology, Shin-Beppu Hospital, Beppu, Japan \\ ${ }^{10}$ Division of Cardiology, Miyazaki Prefectural Nobeoka Hospital, Nobeoka, Japan \\ ${ }^{11}$ Division of Cardiology, Japan Community Health care Organization Kumamoto General Hospital, Yatsushiro, Japan \\ ${ }^{12}$ Division of Cardiology, Japan Labor Health and Welfare Organization Kumamoto Rosai Hospital, Yatsushiro, Japan \\ ${ }^{13}$ Department of Cardiology, National Hospital Organization Kumamoto Medical Center, Kumamoto, Japan \\ ${ }^{14}$ Division of Cardiology, Japanese Red Cross Kumamoto Hospital, Kumamoto, Japan \\ ${ }^{15}$ Division of Cardiology, Kumamoto City Hospital, Kumamoto, Japan \\ ${ }^{16}$ Division of Cardiology, Kumamoto Central Hospital, Kumamoto, Japan \\ ${ }^{17}$ Department of Cardiovascular Medicine, Osaka City University Graduate School of Medicine, Osaka, Japan \\ ${ }^{18}$ National Cerebral and Cardiovascular Center, Suita, Japan
}

Aim: Coronary plaque regression is weak in acute coronary syndrome (ACS) patients with diabetes mellitus (DM). We evaluated whether dual lipid-lowering therapy (DLLT) with ezetimibe and atorvastatin attenuates coronary plaques in ACS patients with DM.

Methods: The prospective, randomized controlled, multicenter PRECISE-IVUS (Plaque Regression with Cholesterol Absorption Inhibitor or Synthesis Inhibitor Evaluated by Intravascular Ultrasound) trial assigned 246 patients undergoing percutaneous coronary intervention to DLLT or atorvastatin monotherapy and evaluated IVUS-derived changes in percent atheroma volume $(\triangle \mathrm{PAV})$, at baseline and 9-12-month follow-up, in 126 ACS cases, including $25 \mathrm{DM}$ patients. The atorvastatin dose was up-titrated to achieve low-density lipoprotein cholesterol (LDL-C) $<70 \mathrm{mg} / \mathrm{dL}$.

Results: In DM patients, the monotherapy group $(n=13)$ and the DLLT group $(n=12)$ showed a similar prevalence of coronary risks and baseline lipid profiles. During the study, the change in LDL-C level was similar between DM and non-DM patients. Compared with non-DM patients, DM patients showed weaker regression of $\triangle \mathrm{PAV}$ by DLLT than those who underwent monotherapy (DM: $-2.77 \pm 3.47 \%$ vs. $-0.77 \pm 2.51 \%, P=0.11$; non-DM: $-2.01 \pm 3.36 \%$ vs. $-0.08 \pm 2.66 \%, P=0.008$ ). The change in LDL-C level was not correlated with $\Delta$ PAV in non-DM patients, but there was significant correlation between the change in LDL-C level and $\triangle$ PAV in DM patients $(r=0.52, P=0.008)$.

Conclusions: ACS patients with DM showed weaker coronary plaque regression than their counterparts. A significant correlation between the change in LDL-C level and $\triangle \mathrm{PAV}$ in DM patients suggested that more intensive lipid-lowering therapy is required in ACS patients with DM.

Key words: Ezetimibe, Statins, Coronary plaque, Diabetes mellitus, Acute coronary syndrome 


\section{Introduction}

Percutaneous coronary intervention (PCI) can improve prognoses in patients with acute coronary syndrome (ACS). However, despite developments in PCI technologies, diabetes mellitus $(\mathrm{DM})^{1)}$ remains a critical cause of poor outcomes after ACS treatment ${ }^{2)}$. Therefore, lifestyle changes or medication are important for secondary prevention in patients with ACS. Large studies have demonstrated that intensive, lipidlowering therapy using statins can reduce the prevalence of cardiovascular events in patients with $\mathrm{ACS}^{3-6)}$. European and American guidelines recommend early, intensive lipid-lowering therapy for ACS management ${ }^{1,7)}$. Studies using intravascular imaging modalities have shown that lipid-lowering therapy can improve the volume and vulnerability of coronary plaques $^{8-13)}$. However, Hiro and colleagues reported that coronary plaque regression is weaker in ACS patients with DM than in those without $\mathrm{DM}^{14)}$.

The randomized Improved Reduction of Outcomes: Vytorin Efficacy International Trial (IMPROVE-IT) demonstrated that dual lipid-lowering therapy (DLLT) using a combination of statin and ezetimibe could reduce the prevalence of cardiovascular events ${ }^{15}$. IMPROVE-IT showed that intensive therapy with ezetimibe to reduce levels of low-density lipoprotein cholesterol (LDL-C) was beneficial against ACS. Furthermore, we reported the benefits of DLLT on coronary plaque regression in the Plaque REgression with Cholesterol absorption Inhibition or Synthesis inhibitor Evaluated by IntraVascular UltraSound (PRECISE-IVUS) trial ${ }^{16)}$. That trial evaluated the effects of an ezetimibe-atorvastatin combination on coronary plaques using intravascular ultrasound (IVUS) in Japanese patients with ACS and stable coronary disease who underwent PCI. In the PRECISEIVUS trial, DLLT, using an ezetimibe-atorvastatin combination, significantly reduced LDL-C levels and coronary plaque volume. In that study, the lower level of LDL-C achieved also correlated with a reduction in the prevalence of cardiovascular events or a change in atheromatous plaque volume, thereby demonstrating "the lower, the better" concept by DLLT using a combination of statins and ezetimibe. However, DLLT's benefit on coronary plaques in ACS patients with DM has not been investigated.

We investigated whether DLLT, using an ezetimibe-atorvastatin combination, attenuated coronary plaque development in ACS patients with DM. In addition, several clinical factors, e.g., sex, hypertension, and obesity, are reportedly associated with coronary plaque development ${ }^{17-19)}$. However, the determinant factors related to coronary plaque regression in ACS patients with DM are not known, a knowledge gap that we attempted to fill.

\section{Methods}

\section{Design of the PRECISE-IVUS Trial}

The present study is a sub-analysis of the PRECISE-IVUS trial. The detailed design of the PRECISE-IVUS trial has been published ${ }^{16}$. In brief, PRECISE-IVUS was a prospective, randomized, controlled, assessor-blind, multicenter trial to evaluate the benefit of an ezetimibe-atorvastatin combination on coronary plaque volume, measured by IVUS, in patients with coronary artery disease. Eligible patients (30-85 years of age and LDL-C level upon study enrollment $>100 \mathrm{mg} / \mathrm{dL}$ ) were assigned randomly to receive atorvastatin alone or an ezetimibe-atorvastatin combination at $10 \mathrm{mg}$ every day (DLLT group) after having undergone successful IVUS-guided PCI to treat ACS or stable angina pectoris. The atorvastatin dose was up-titrated to achieve the target level of LDL-C $(<70 \mathrm{mg} / \mathrm{dL})$. Biomarkers, including lipid profiles, and periodic medical examination were measured 3, 6, and 9-12 months after enrollment. Serial volumetric IVUS was carried out at baseline and 9-12 months to evaluate coronary plaque changes. The PRECISE-IVUS trial enrolled 246 patients, and 126 patients were enrolled as the ACS cohort. This substudy evaluated these 126 patients with ACS. The sub-study flowchart is shown in Fig. 1 . An equal number of patients were assigned to the DLLT group and atorvastatin monotherapy group. Fourteen patients were excluded from the atorvastatin monotherapy group ( 2 patients withdrew consent, and 12 did not complete endpoint assessment) and 12 patients were excluded from the DLLT group (1 patient withdrew consent, and 11 did not complete endpoint assessment). Finally, 49 and 51 patients had full datasets for atorvastatin monotherapy and DLLT, respectively. The monotherapy group had 13 patients with DM, and the DLLT group contained 12 patients with DM.

The present study complied with the Declaration of Helsinki with respect to clinical investigations. The study protocol was approved by the Human Ethics

Address for correspondence: Koichiro Fujisue, Department of Cardiovascular Medicine, Graduate School of Medical Sciences and Center for Metabolic Regulation of Healthy Aging (CMHA), Kumamoto University, 1-1-1 Honjo, Chuo-ku, Kumamoto 860-8556, Japan. E-mail: fujisues@kumamoto-u.ac.jp

Received: December 13, 2019 Accepted for publication: March 13, 2020

Copyright@2021 Japan Atherosclerosis Society

This article is distributed under the terms of the latest version of CC BY-NC-SA defined by the Creative Commons Attribution License. 


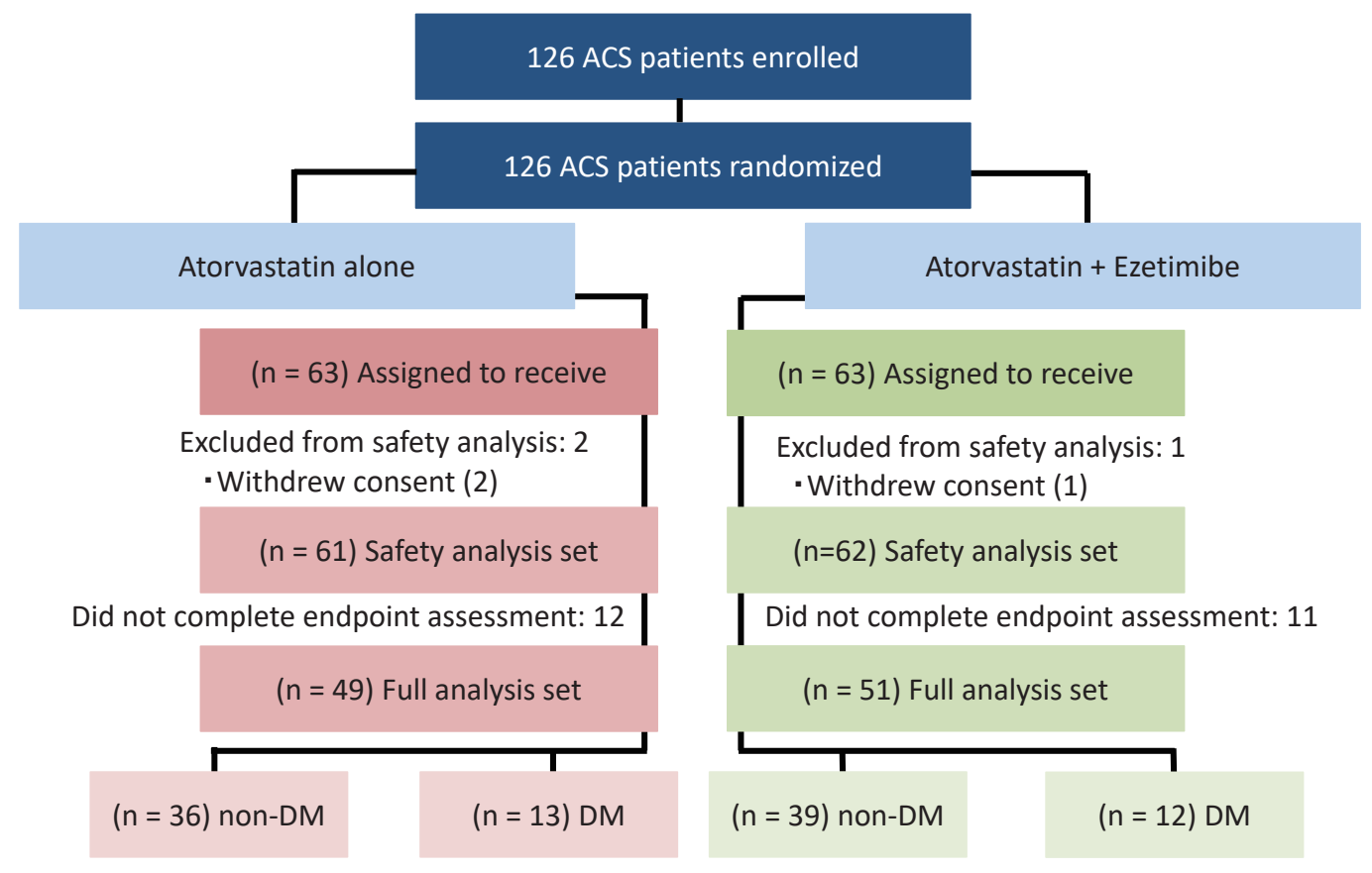

Fig. 1. Flowchart of the sub-study of the PRECISE-IVUS trial

ACS, acute coronary syndrome; DM, diabetes mellitus

Review Committee of Kumamoto University. The study was conducted in accordance with the guidelines of the participating institution's ethics committee. Written informed consent was obtained from all ACS patients until $24 \mathrm{~h}$ after PCI.

\section{Definition of ACS}

ACS was defined as ST-elevation myocardial infarction (STEMI), non-STEMI, or unstable angina pectoris. MI was diagnosed by increased levels of cardiac biomarkers in plasma (creatine kinase-MB or cardiac troponin) above the 99th percentile of the upper limit of the normal range, together with evidence of myocardial ischemia and at least one of the following symptoms: electrocardiographic changes (new ST-T changes, left bundle branch block, or pathologic Q wave) or imaging evidence of new viable myocardial loss or a new abnormality in regional wall motion ${ }^{20)}$. Unstable angina pectoris was diagnosed by new or accelerating myocardial ischemia symptoms accompanied by new ischemic ST-T-wave changes.

\section{Definition of DM}

The diagnosis of DM was made according to the diagnostic criteria from the Japanese Diabetes Society. We defined DM when patients had a history of DM diagnoses or were taking any hypoglycemic medications. If this information were unavailable, the diagnosis of DM was made when "diabetic type" (fasting plasma glucose concentration $\geq 126 \mathrm{mg} / \mathrm{dL}$, fasting plasma glucose concentration $\geq 126 \mathrm{mg} / \mathrm{dL}$, 2 -h plasma glucose concentration $\geq 200 \mathrm{mg} / \mathrm{dL}$ during a 75 -g oral glucose tolerance test, casual plasma glucose concentration $\geq 200 \mathrm{mg} / \mathrm{dL}$, or HbA1c $\geq 6.5 \%$ ) was confirmed on two or more occasions examined on separate days; a single plasma glucose test meeting criteria for "diabetic type," when any of the following three conditions were met: (1) typical symptoms of diabetes (thirst, polydipsia, polyuria, and weight loss), (2) $\mathrm{HbA}_{1 \mathrm{c}} \geq 6.5 \%$, or (3) diabetic retinopathy.

\section{IVUS Imaging for Coronary Plaque Analyses}

Details of recording of IVUS images have been described ${ }^{16)}$. An IVUS catheter was inserted into the target vessel for PCI as distal as possible to safely obtain the longest possible target segment for analyses. Then, it was pulled back at $0.5 \mathrm{~mm} / \mathrm{s}$ automatically after intracoronary injection of nitroglycerin (0.1-0.2 $\mathrm{mg})$. The target segment to be monitored was determined at a non-PCI site $(>5 \mathrm{~mm}$ proximal or distal from the PCI site), with a reproducible fiduciary index (usually a side branch), as the beginning and ending of the segments to be analyzed. IVUS images were analyzed by two independent, experienced observers who were unaware of the treatment allocation and temporal sequence of paired images, as recommended by expert consensus ${ }^{21)}$. Based on expert consensus, the primary endpoint was the absolute change in percent 
atheroma volume $(\triangle \mathrm{PAV})^{21)}$.

\section{Statistical Analyses}

Data are the mean \pm standard deviation for variables with a normal distribution. Data with skewed distributions are expressed as the median with interquartile range. Continuous variables among groups were compared using the unpaired Student's $t$-test or Mann-Whitney $U$-test, as appropriate. Continuous variables between baseline and follow-up were compared by one-sample Student's $t$-tests or the Wilcoxon signed rank test according to their distributions. Categorical variables were compared using the chi-square test or Fisher's exact test. The relationship between the absolute change in PAV and several biomarkers (including these for cholesterol absorption) was evaluated using a simple regression analysis. Pearson's correlation coefficient $(r)$ was used to evaluate the association between PAV and biomarkers. Also, Spearman's rank correlation coefficient $(\rho)$ was used if the variables did not have a normal distribution. $P<0.05$ was considered significant. Statistical analyses were carried out using SPSS v25 (IBM, Armonk, USA).

\section{Results}

\section{Baseline Characteristics of Enrolled Patients}

Table 1 shows the baseline characteristics of enrolled patients. In DM patients, the monotherapy group and DLLT group showed a similar prevalence of coronary risks, baseline lipid profiles, and medications. Forty-eight patients with ACS have been treated with statins; the statins were up-titrated according to the study protocol in all patients. Twelve-percent of the patients were receiving insulin. In non-DM patients, the percentage of current smokers was significantly higher in the monotherapy group than in the DLLT group. The baseline levels of campesterol and sitosterol (markers of cholesterol absorption) were significantly higher in the DLLT group than in the monotherapy group (non-DM: campesterol, 4.7 (3.6 to 6.4$)$ vs. $3.4(2.9$ to 4.5$) \mu \mathrm{g} / \mathrm{dL}, P=0.04$; DM: sitosterol, $2.7(1.9$ to 3.3$)$ vs. $1.8(1.5$ to 2.3$) \mu \mathrm{g} / \mathrm{dL}, P=$ $0.01)$.

\section{Laboratory Data during the Study Period}

Table 2 and Supplemental Table 1 show the percent change of laboratory data during the study period. HbA1c level did not change significantly between the monotherapy group and the DLLT group in $\mathrm{DM}$ and non-DM patients. The percent change in the LDL-C level is shown in Table 2 and Fig. 2. The serum level of LDL-C was reduced in all groups. In non-DM patients, the percent change in the LDL-C level significantly decreased in the DLLT group compared with that in the monotherapy group (DLLT group, $-23.0 \pm 23.2 \%$ vs. monotherapy group, -23.0 $\pm 23.2 \%, P<0.001)$. The percent change in the LDL-C level in DM patients tended to be reduced by DLLT, but it was not significant (DLLT group, -42.9 $\pm 13.8 \%$ vs. monotherapy group, $-29.2 \pm 30.6 \%, P=$ 0.16) (Fig. 2A). The percent change in the apolipoprotein (Apo)A1/ApoB ratio was reduced significantly by DLLT in the non-DM and DM group. The levels of campesterol and sitosterol were increased by monotherapy in the non-DM and DM group but were reduced by DLLT. The level of lathosterol (marker of cholesterol synthesis) was reduced in all groups (Table 2).

\section{$\triangle \mathrm{PAV}$}

Compared with non-DM patients, DM patients showed weaker regression of $\triangle \mathrm{PAV}$ in the DLLT group than in the monotherapy group (non-DM: DLLT group, $-2.01 \pm 3.36 \%$ vs. monotherapy group, $-0.08 \pm 2.66 \%, P=0.008$; DM: DLLT group, -2.77 $\pm 3.47 \%$ vs. monotherapy group, $-0.77 \pm 2.51 \%, P=$ 0.11) (Table 2, Fig. 2B). The total atheroma volume showed similar results between non-DM and DM patients (non-DM: DLLT group, $-9.02 \pm 14.71 \%$ vs. monotherapy group, $0.93 \pm 8.67 \%, P=0.001$; DM: DLLT group, $-1.60 \pm 14.62 \%$ vs. monotherapy group, $-7.54 \pm 7.04 \%, P=0.20)$. In patients with $\mathrm{DM}$, vessel volume and lumen volume tended to be reduced in the monotherapy group; however, those were inhibited in the DLLT group, although they were not significant (Table 2).

Relationship between Coronary Plaque Regression and Biomarker Levels

Table 3 shows the relationship between lipid profiles and $\triangle \mathrm{PAV}$. There was no correlation between the percent change in the HbA1c level and $\triangle \mathrm{PAV}$ (non-DM, $r=0.11, P=0.42$; DM, $r=0.14, P=0.51$, respectively) (Fig. 3A, B). The LDL-C level at 9-12month follow-up was significantly correlated with $\Delta$ PAV in DM patients $(r=0.52, P=0.008)$, but not in non-DM patients $(r=0.12, P=0.31)$ (Fig. 3A). Furthermore, the percent change in the LDL-C level was significantly correlated with PAV in DM patients $(r=$ $0.44, P=0.03)$, but not in non-DM patients $(r=0.13$, $P=0.25)$ (Fig. 3B). The percent change in the ApoB level and ApoB/ApoA1 ratio was significantly correlated with $\triangle \mathrm{PAV}$ in DM patients $(\rho=0.41, P=0.04$, and $\rho=0.52, P=0.007$, respectively) (Table 3 ), but not in non-DM patients $(\rho=0.13, P=0.27$, and $\rho$ $=0.012, P=0.92$, respectively) (Table 3 ). The percent change in the levels of campesterol and sitosterol was 
Table 1. Baseline characteristics of enrolled patients

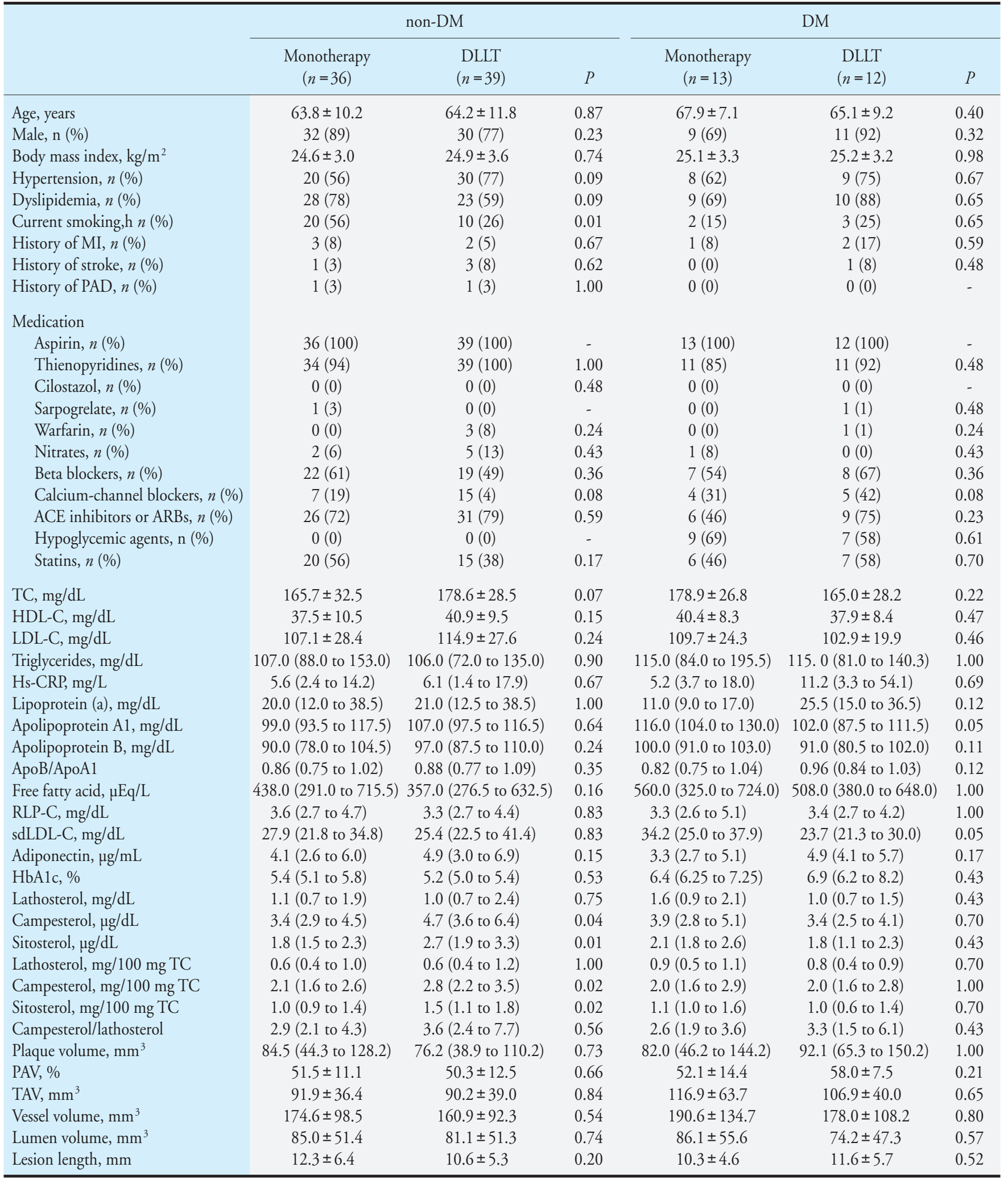

Data are the $n(\%)$, mean \pm SD, or median (IQR). DM, diabetes mellitus; DLLT, dual lipid-lowering therapy with atorvastatin and ezetimibe; MI, myocardial infarction; PAD, peripheral artery disease; ACE, angiotensin-converting enzyme; ARB, angiotensin receptor blocker; TC, total cholesterol; HDL-C, high-density lipoprotein-cholesterol; LDL-C, low-density lipoprotein-cholesterol; Hs-CRP, high sensitive C-reactive protein; RLP$\mathrm{C}$, remnant like particle-cholesterol; sdLDL-C, small dense low-density lipoprotein-cholesterol; PAV, percent atheroma volume; TAV, total atheroma volume. 
Table 2. Percent change in laboratory data during the study period

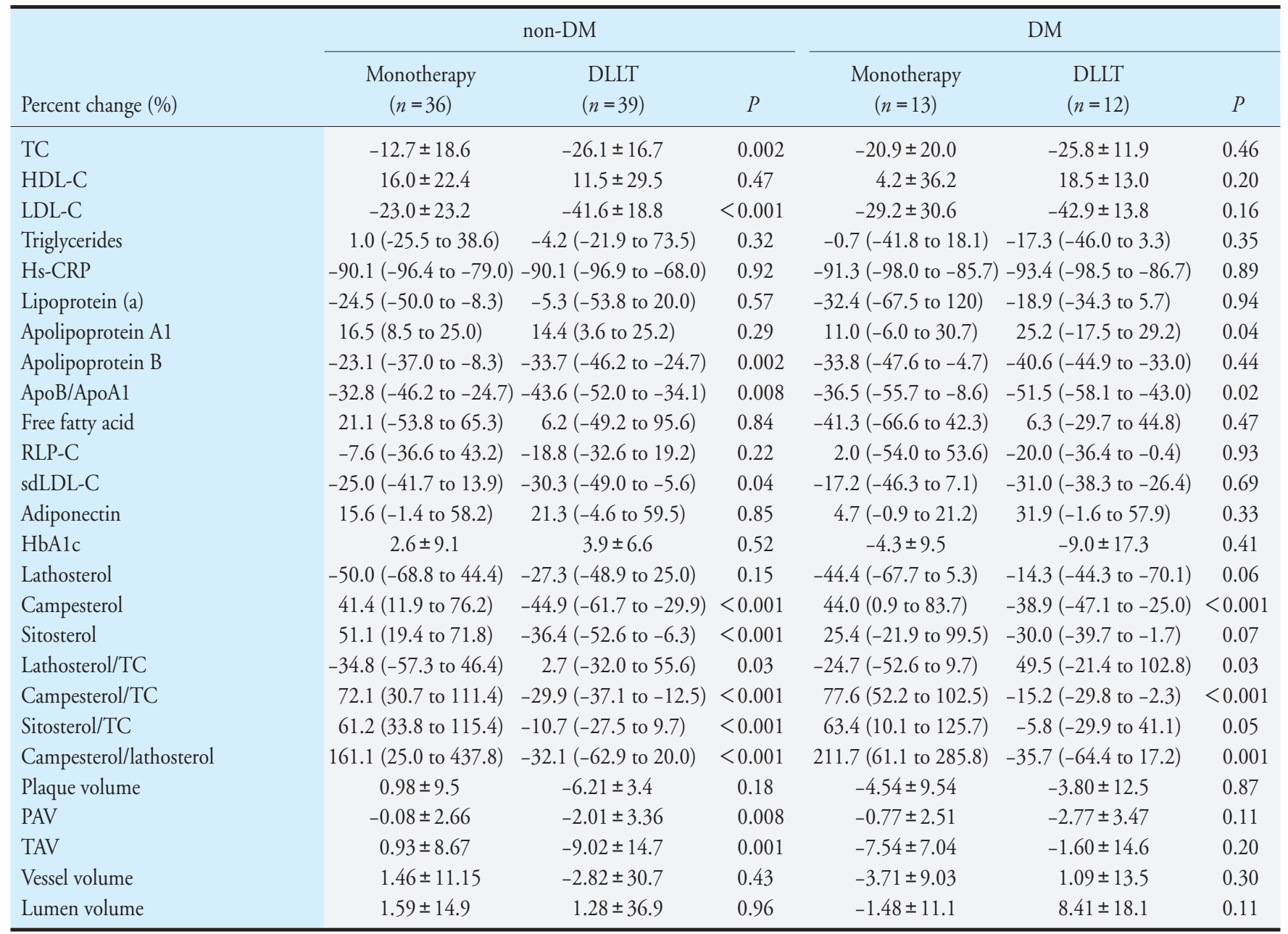

See Table 1 for abbreviations.

significantly correlated with $\triangle \mathrm{PAV}$ in non-DM patients $(\rho=0.34, P=0.004$, and $\rho=0.31, P=0.009$, respectively), but not in DM patients. The baseline levels of campesterol and sitosterol were not correlated with $\triangle \mathrm{PAV}$.

\section{Discussion}

This sub-study of the PRECISE-IVUS trial showed that coronary plaque tended to be reduced by intensive lipid-lowering therapy, but it was significantly weak in ACS patients with DM even if they underwent DLLT. $\triangle$ PAV seemed to be reduced in the DM-DLLT group compared with non-DM monotherapy group. However, there was no statistical difference between non-DM monotherapy and DM$\operatorname{DLLT}(P=0.06)$ by one-way analysis of variance with repeated measures followed by a Bonferroni multiple comparison adjustment. This result suggests that DLLT could be more effective to attenuate $\triangle \mathrm{PAV}$ than monotherapy even in DM patients, but this might not be enough for LDL-C management in DM patients. Baseline characteristics, coronary plaque volume, vessel size, and lesion length were similar between the monotherapy group and DLLT group in ACS patients with DM. The reduction in the LDL-C level was similar between patients with and without DM. In patients with DM, DLLT reduced the LDL-C level $<70 \mathrm{mg} / \mathrm{dL}$, as recommended in guidelines. The HbA1c level did not increase through the follow-up period. Therefore, poor control of LDL-C or glucose levels was not related to weaker regression of coronary plaques in patients with DM.

Our study revealed other important results. Although $\triangle \mathrm{PAV}$ in patients with $\mathrm{DM}$ was not significantly different between the monotherapy group and DLLT group, a significant correlation between the percent change in the LDL-C level and $\triangle \mathrm{PAV}$ was observed in DM patients, but not in non-DM patients. Baseline characteristics were similar between 
A

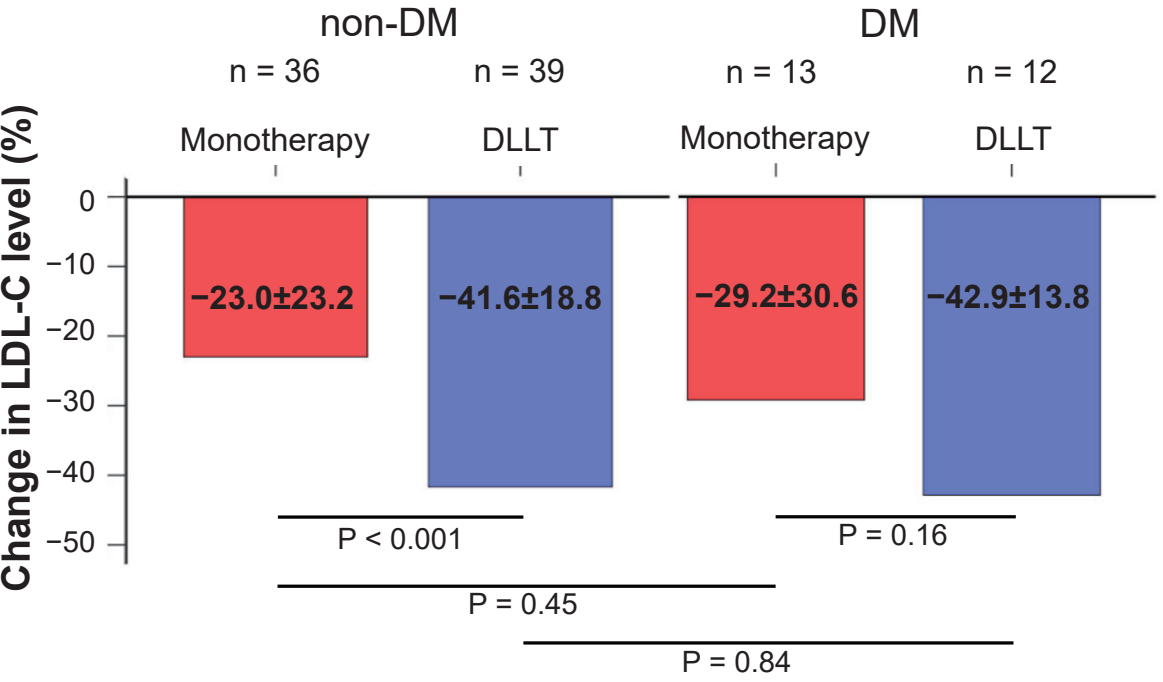

B

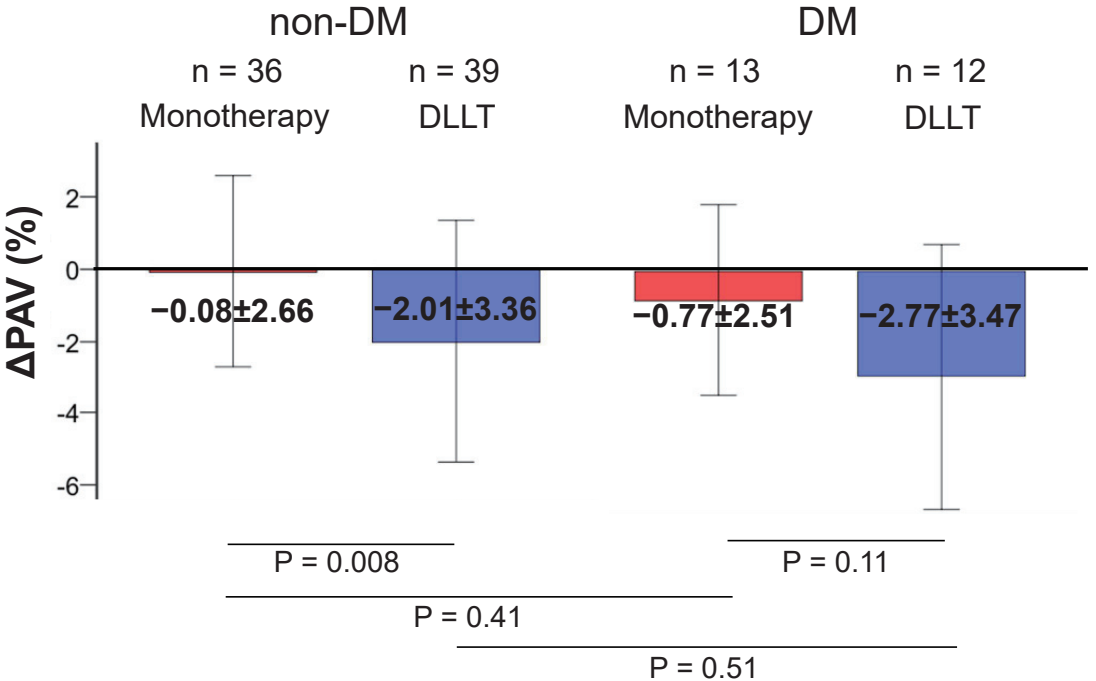

Fig. 2. Percent change in levels of hemoglobin A1c, low-density lipoprotein cholesterol, and percent coronary plaque volume between the monotherapy group and dual lipid-lowering group in ACS patients with and without diabetes mellitus

In non-DM ${ }^{1)}$ patients, the percent change in the LDL-C level was reduced significantly in the dual lipid-loweringtherapy (DLLT) group compared with the monotherapy group (A). The percent change in the LDL-C level in DM patients tended to be reduced by DLLT, but this reduction was not significant (A). Compared with non-DM patients, DM patients showed weaker regression of the change in the percent atheroma volume ( $\triangle \mathrm{PAV})(\mathrm{B})$.

DM and non-DM patients with ACS except for the baseline levels of campesterol and sitosterol, which are cholesterol absorption markers. In non-DM patients, markers of cholesterol absorption were associated with $\Delta$ PAV. Strandberg et al. associated that higher cholesterol absorption with a higher prevalence of cardiovascular events ${ }^{22)}$. Statins increase cholesterol absorption $^{23)}$, which could be associated with accelerated atherosclerosis ${ }^{24)}$. Therefore, increased levels of cholesterol absorption markers may have attenuated the benefit of the reduced LDL-C level on $\triangle \mathrm{PAV}$ in our study, but this phenomenon was not observed in DM patients. It has been reported that cholesterol metabolism is disturbed so that cholesterol synthesis is elevated, while cholesterol absorption efficiency is reduced in patients with $\mathrm{DM}^{22}$. Ezetimibe's efficacy might be reduced under the condition of decreased cholesterol absorption. Furthermore, the percent change of lathosterol/TC ratio, which is the cholesterol synthesis marker, was increased in the DLLT group in DM patients compared with non-DM patients $(P<0.001)$. Therefore, our observation of cholesterol metabolism markers may suggest a different plaque regression mechanism between DM and 
Table 3. Relationship between lipid profiles and $\triangle \mathrm{PAV}$

\begin{tabular}{lccccc}
\hline & \multicolumn{2}{c}{ non-DM } & \multicolumn{3}{c}{ DM } \\
\cline { 2 - 3 } Percent change during follow-up & $r$ & $P$ & & $r$ & $P$ \\
\hline HbA1c, \% & 0.11 & 0.42 & & 0.14 & 0.51 \\
LDL-C, \% & 0.13 & 0.25 & & 0.44 & 0.03 \\
\hline & $\rho$ & $P$ & & $\rho$ & $P$ \\
\hline Lipoprotein (a), \% & -0.07 & 0.59 & & 0.09 & 0.71 \\
ApoA1, \% & 0.05 & 0.68 & & -0.11 & 0.62 \\
ApoB, \% & 0.13 & 0.27 & & 0.41 & 0.04 \\
ApoB/ApoA1, \% & 0.012 & 0.92 & & 0.52 & 0.007 \\
RLP-C, \% & 0.15 & 0.22 & & 0.12 & 0.57 \\
sdLDL-C, \% & 0.18 & 0.13 & & 0.30 & 0.15 \\
Lathosterol/TC, \% & -0.067 & 0.58 & & -0.18 & 0.38 \\
Campesterol/TC, \% & 0.34 & 0.004 & & 0.23 & 0.27 \\
Sitosterol/TC, \% & 0.31 & 0.009 & & 0.29 & 0.15 \\
\hline
\end{tabular}

See Table 1 for abbreviations.

Furthermore, the percent change in the LDL-C level was significantly correlated with PAV in patients with $\mathrm{DM}(r=0.04, P=0.03)$, but not in non-DM patients $(r=0.13, P=0.25$, Fig. $3 \mathrm{~A}, \mathrm{~B})$.

A

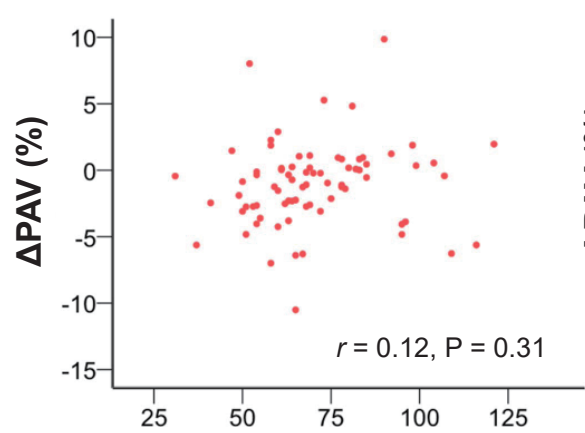

LDL-C, follow-up (mg/dL) non-DM

B

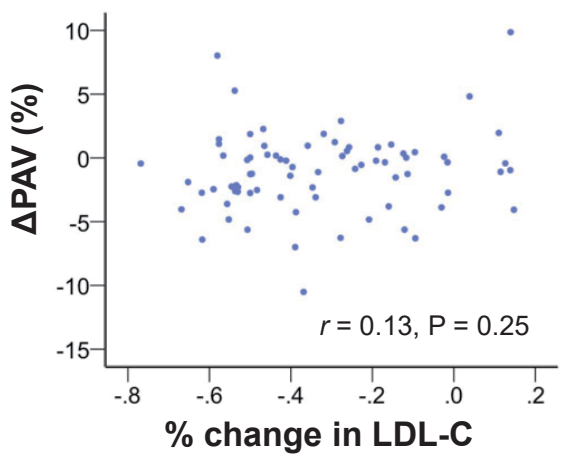

non-DM

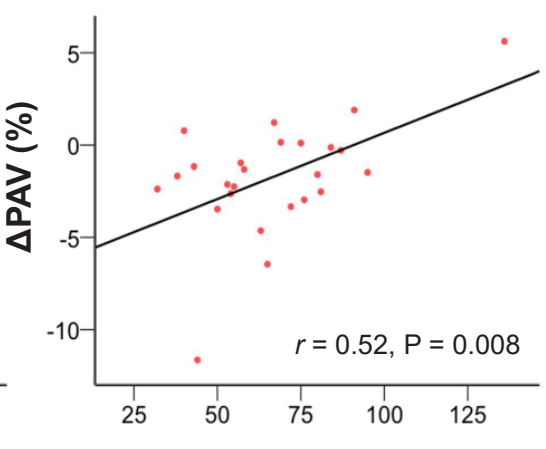

LDL-C, follow-up (mg/dL)

DM

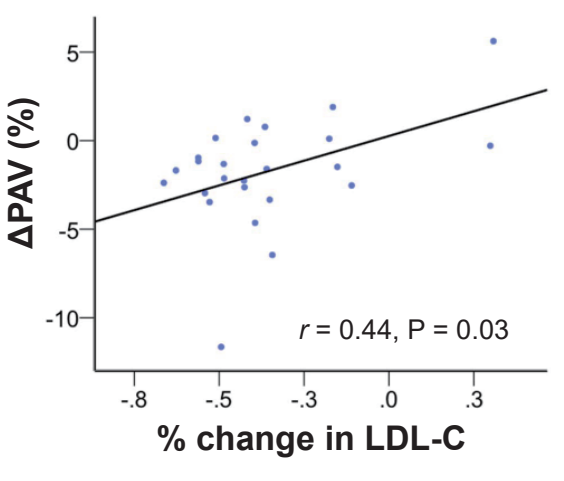

DM

Fig.3. Relationship between the level of low-density lipoprotein cholesterol and change in percent atheroma volume

Relationship between change in low-density lipoprotein cholesterol (LDL-C) level at follow-up (A), percent change in the level of LDL-C (B), and change in percent atheroma volume ( $\triangle \mathrm{PAV})$ in ACS patients with and without DM. 
non-DM patients. Furthermore, vessel volume and lumen volume tended to be reduced in the monotherapy group in patients with DM, but these features tended to be increased in the DLLT group; these changes were not observed in non-DM patients. These results suggest that people with DM and individuals not suffering from DM may have different coronary plaque characteristics and pathologic mechanisms in coronary plaque regression.

Scholars using IVUS have reported that ACS patients with DM have greater coronary plaque burden and necrotic core volume than those without $\mathrm{DM}^{25,26)}$. Pathology studies have also shown that the coronary plaques of patients with DM have large necrotic cores and accumulate macrophages and $\mathrm{T}$ cells, which reflect increased inflammation and which are associated with atherosclerosis progression and coronary plaque vulnerability in $\mathrm{DM}^{27,28)}$. Those studies supported our hypothesis of different coronary plaque characteristics.

A systematic review by Williams et al. postulated a coronary plaque regression mechanism. They stated that extensive lowering of the ApoB level can activate reverse lipid transportation from atheromatous plaques toward the liver ${ }^{29)}$. Possible mechanisms for plaque regression which Williams et al. suggested are decreased retention of ApoB-containing lipoprotein within the arterial wall, efflux of cholesterol and other toxic lipids from plaques, emigration of foam cells out of the arterial wall, and migration of healthy phagocytes that remove necrotic debris. Reduction of the necrotic core and the number of inflammatory cells within atheromatous plaques are crucial for regression of coronary plaques in patients with DM. For this to occur, more intensive lowering of levels of $A p o B$ and cholesterol would be required. Further basic and clinical evaluation is required to explain these mechanisms.

Hiro et al. reported similar results when evaluating coronary plaque changes using IVUS in 252 ACS patients treated with pitavastatin or atorvastatin ${ }^{14}$. In their study, coronary plaque regression was weaker in $\mathrm{DM}$ patients than in non-DM patients. In DM patients with baseline $\mathrm{HbA1c} \geq 7.0 \%$, there were significant correlations between the coronary plaque volume and LDL-C level at 8-12-month follow-up and the percent change of this parameter during the study period; such effects were not observed in DM patients with baseline $\mathrm{HbAlc}<7.0 \%$. They suggested that glycemic control might be essential for coronary plaque regression in ACS patients with DM. Furthermore, they reported significant correlations between $\triangle \mathrm{PAV}$ and the percent changes of other lipid parameters (e.g., total cholesterol, remnant-like particle cholesterol, non-high-density lipoprotein cholesterol,
ApoB) in DM patients, but not in non-DM patients.

In our study, the change in the HbAlc level was not correlated with $\triangle \mathrm{PAV}$. LDL-C dependency, rather than glycemic control, seemed to be important. In addition, the percent change in the ApoB level and ApoB/ApoA1 ratio was significantly correlated with $\Delta$ PAV in DM patients, but not in non-DM patients. $A p o B$ is a main component of chylomicron and verylow-density lipoprotein. Catabolism of chylomicron and very-low-density lipoprotein is attenuated in DM, so atherogenic lipoproteins tend to accumulate. This effect can lead to the development of more lipid-rich coronary plaques in patients with DM. Regression of the lipid-rich components in coronary plaques could depend on reduced LDL-C and ApoB levels in patients with DM. Therefore, more intensive therapy to reduce LDL-C and ApoB levels would be required in patients with DM.

\section{Limitations}

Our study had two main limitations. First, this sub-study was a retrospective analysis, and the baseline characteristics were not matched completely, primarily because of the small sample size (especially for DM patients). Although there was no statistical difference in $\triangle \mathrm{PAV}$ in DM patients, absolute regression appears to be similar between DM and non-DM patients in Fig. 2B. Patients with DM might not have a significant difference due to small sample size, so the results should be interpreted carefully. Second, DM was mainly diagnosed according to medical history or laboratory data upon hospital admission. The glucose tolerance test was not carried out in some patients, so DM screening was suboptimal. Therefore, a larger prospective, randomized control trial would be required to confirm our results.

\section{Conclusions}

ACS patients with DM showed weaker regression of coronary plaques than non-DM patients. However, the percent change in levels of LDL-C and $A p o B$ was correlated significantly to $\triangle \mathrm{PAV}$ in $\mathrm{DM}$ patients, suggesting that more intensive lipid-lowering therapy with an ezetimibe-statin combination would be beneficial in ACS patients with DM.

\section{Notice of Grant Supports}

This study was supported in part by a Grant-inAid for Young Scientists B (22790713, 24790769 to K.T, and 17K17956 to K.F.), Grant-in-Aid for Young Scientists (19K1750 to K.F.) and Grant-in-aid for Sci- 
entific Research C (26461075, and $18 \mathrm{~K} 08110$ to K. T.) from the Ministry of Education, Science, and Culture, Japan.

\section{Acknowledgements}

We thank Akiyo Kikuchi and Yuko Kuratsu for their secretarial assistance, as well as Michiyo Saito for technical assistance in angiographic and the acquisition and measurement of data obtained using intravascular ultrasound. We thank Arshad Makhdum, $\mathrm{PhD}$, from Edanz Group (www.edanzediting.com/ac) for editing a draft of this manuscript.

\section{Fundings}

This study was supported in part by a Grant-inAid for Young Scientists B (22790713, 24790769 to K.T, and 17 K17956 to K.F.), Grant-in-Aid for Young Scientists (19K1750 to K.F.) and Grant-in-aid for Scientific Research C (26461075, and 18K08110 to K. T.) from the Ministry of Education, Science, and Culture, Japan.

\section{Conflicts of Interests}

KS has received significant research grant support from Daiichi-Sankyo, Co., Ltd. Japan, outside the submitted work. KK has received significant research grant support from Bayer, Yakuhin, Ltd., Daiichi Sankyo Co., Ltd., Novartis Pharma AG., and SBI, Pharma Co., Ltd., and has received Honoraria from Bayer Yakuhin, Ltd. and Daiichi Sankyo Co., Ltd., outside the submitted work. HO has received grants from Astellas Pharma Incorporated, personal fees from Astra Zeneca Kabushiki Kaisha, grants and personal fees from Bayer Yakuhin, Limited, personal fees from Boehringer Ingelheim Japan, grants and personal fees from Bristol-Myers Squibb Company, grants and personal fees from Daiichi Sankyo Company, Limited, grants from Dainippon Sumitomo Pharma Company, Limited, grants and personal fees from Eisai Company, Limited, personal fees from Kowa Company, Limited, personal fees from Kyowa Hakko Kirin Company, Limited, grants and personal fees from Mitsubishi Tanabe Pharma, grants and personal fees from MSD Kabushiki Kaisha, grants from Novartis Pharma Kabushiki Kaisha, grants from Otsuka Pharmaceutical Company, Limited, grants and personal fees from Pfizer Japan Incorporated, grants and personal fees from Sanofi Kabushiki Kaisha, grants from Shionogi Company, Limited, grants and personal fees from Takeda Pharmaceutical Company, Limited, grants and personal fees from Teijin Pharma
Company Limited, grants from Genzyme Japan Kabushiki Kaisha, grants from Kissei Pharmaceutical Company Limited, grants from Abbott Vascular Japan, grants from Boston Scientific Japan Kabushiki Kaisya, grants from Fukuda Denshi Company, Limited, grants from Johnson \& Johnson, grants from Medtronic Japan Company Limited, grants from Nihon Kohden, and grants from Terumo, outside the submitted work. All other authors have reported that they have no relationships relevant to the contents of this paper to disclose.

\section{References}

1) Catapano AL, Graham I, De Backer G, Wiklund O, Chapman MJ, Drexel H, Hoes AW, Jennings CS, Landmesser U, Pedersen TR, Reiner Z, Riccardi G, Taskinen MR, Tokgozoglu L, Verschuren WMM, Vlachopoulos C, Wood DA, Zamorano JL and Cooney MT: 2016 ESC/ EAS Guidelines for the Management of Dyslipidaemias. Eur Heart J, 2016; 37: 2999-3058

2) Suzuki K, Ishikawa T, Mutoh M, Sakamoto H, Kubota T, Ogawa T, Mori C, Hashimoto K, Komukai K and Yoshimura M: Midterm angiographic outcomes with sirolimus- and everolimus-eluting stents for small vessels in diabetic patients: propensity-score-matched comparisons in three different vessel diameters. Cardiovasc Interv Ther, 2018; 33: 205-216

3) Schwartz GG, Olsson AG, Ezekowitz MD, Ganz P, Oliver MF, Waters D, Zeiher A, Chaitman BR, Leslie S and Stern T: Effects of atorvastatin on early recurrent ischemic events in acute coronary syndromes: the MIRACL study: a randomized controlled trial. JAMA, 2001; 285: 17111718

4) Ray KK, Cannon CP, McCabe CH, Cairns R, Tonkin AM, Sacks FM, Jackson G and Braunwald E: Early and late benefits of high-dose atorvastatin in patients with acute coronary syndromes: results from the PROVE ITTIMI 22 trial. J Am Coll Cardiol, 2005; 46: 1405-1410

5) Baigent C, Keech A, Kearney PM, Blackwell L, Buck G, Pollicino C, Kirby A, Sourjina T, Peto R, Collins R and Simes R: Efficacy and safety of cholesterol-lowering treatment: prospective meta-analysis of data from 90,056 participants in 14 randomised trials of statins. Lancet, 2005; 366: $1267-1278$

6) Cho J, Park IB, Lee K, Ahn TH, Park WB, Kim JH, Ahn $\mathrm{Y}$, Jeong $\mathrm{MH}$ and Lee $\mathrm{DH}$ : Statin has more protective effects in AMI patients with higher plasma BNP or NTproBNP level, but not with lower left ventricular ejection fraction. J Cardiol, 2018; 71: 375-381

7) Grundy SM, Stone NJ, Bailey AL, Beam C, Birtcher KK, Blumenthal RS, Braun LT, de Ferranti S, Faiella-Tommasino J, Forman DE, Goldberg R, Heidenreich PA, Hlatky MA, Jones DW, Lloyd-Jones D, Lopez-Pajares N, Ndumele CE, Orringer CE, Peralta CA, Saseen JJ, Smith SC, Jr., Sperling L, Virani SS and Yeboah J: 2018 AHA/ACC/ AACVPR/AAPA/ABC/ACPM/ADA/AGS/APhA/ASPC/ NLA/PCNA Guideline on the Management of Blood Cholesterol: A Report of the American College of Cardi- 
ology/American Heart Association Task Force on Clinical Practice Guidelines. J Am Coll Cardiol, 2019; 73: e285-e350

8) Okazaki S, Yokoyama T, Miyauchi K, Shimada K, Kurata T, Sato $\mathrm{H}$ and Daida $\mathrm{H}$ : Early statin treatment in patients with acute coronary syndrome: demonstration of the beneficial effect on atherosclerotic lesions by serial volumetric intravascular ultrasound analysis during half a year after coronary event: the ESTABLISH Study. Circulation, 2004; 110: 1061-1068

9) Nissen SE, Tuzcu EM, Schoenhagen P, Brown BG, Ganz P, Vogel RA, Crowe T, Howard G, Cooper CJ, Brodie B, Grines CL and DeMaria AN: Effect of intensive compared with moderate lipid-lowering therapy on progression of coronary atherosclerosis: a randomized controlled trial. JAMA, 2004; 291: 1071-1080

10) Kawasaki M, Sano K, Okubo M, Yokoyama H, Ito $Y$, Murata I, Tsuchiya K, Minatoguchi S, Zhou X, Fujita H and Fujiwara $\mathrm{H}$ : Volumetric quantitative analysis of tissue characteristics of coronary plaques after statin therapy using three-dimensional integrated backscatter intravascular ultrasound. J Am Coll Cardiol, 2005; 45: 1946-1953

11) Sipahi I, Nicholls SJ, Tuzcu EM and Nissen SE: Coronary atherosclerosis can regress with very intensive statin therapy. Cleve Clin J Med, 2006; 73: 937-944

12) Hiro T, Kimura T, Morimoto T, Miyauchi K, Nakagawa Y, Yamagishi M, Ozaki Y, Kimura K, Saito S, Yamaguchi T, Daida $\mathrm{H}$ and Matsuzaki M: Effect of intensive statin therapy on regression of coronary atherosclerosis in patients with acute coronary syndrome: a multicenter randomized trial evaluated by volumetric intravascular ultrasound using pitavastatin versus atorvastatin (JAPAN-ACS [Japan assessment of pitavastatin and atorvastatin in acute coronary syndrome] study). J Am Coll Cardiol, 2009; 54: 293-302

13) Otake H, Tanimura K, Sugizaki Y, Toba T, Kawamori H, Shinke T and Hirata K-i: Effect of Alirocumab and Rosuvastatin or Rosuvastatin Alone on Lipid Core Plaque in Coronary Artery Disease Seen on Near-Infrared Spectroscopy Intravascular Ultrasound (ANTARES). Circulation Reports, 2019; 1: 107-111

14) Hiro T, Kimura T, Morimoto T, Miyauchi K, Nakagawa Y, Yamagishi M, Ozaki Y, Kimura K, Saito S, Yamaguchi T, Daida $\mathrm{H}$ and Matsuzaki $\mathrm{M}$ : Diabetes mellitus is a major negative determinant of coronary plaque regression during statin therapy in patients with acute coronary syndrome--serial intravascular ultrasound observations from the Japan Assessment of Pitavastatin and Atorvastatin in Acute Coronary Syndrome Trial (the JAPAN-ACS Trial). Circ J, 2010; 74: 1165-1174

15) Cannon CP, Blazing MA, Giugliano RP, McCagg A, White JA, Theroux P, Darius H, Lewis BS, Ophuis TO, Jukema JW, De Ferrari GM, Ruzyllo W, De Lucca P, Im K, Bohula EA, Reist C, Wiviott SD, Tershakovec AM, Musliner TA, Braunwald E and Califf RM: Ezetimibe Added to Statin Therapy after Acute Coronary Syndromes. N Engl J Med, 2015; 372: 2387-2397

16) Tsujita K, Sugiyama S, Sumida H, Shimomura $H$, Yamashita T, Yamanaga K, Komura N, Sakamoto K, Oka H, Nakao K, Nakamura S, Ishihara M, Matsui K, Sakaino N, Nakamura N, Yamamoto N, Koide S, Matsumura T,
Fujimoto K, Tsunoda R, Morikami Y, Matsuyama K, Oshima S, Kaikita K, Hokimoto S and Ogawa H: Impact of Dual Lipid-Lowering Strategy With Ezetimibe and Atorvastatin on Coronary Plaque Regression in Patients With Percutaneous Coronary Intervention: The Multicenter Randomized Controlled PRECISE-IVUS Trial. J Am Coll Cardiol, 2015; 66: 495-507

17) Nicholls SJ, Tuzcu EM, Crowe T, Sipahi I, Schoenhagen P, Kapadia S, Hazen SL, Wun CC, Norton M, Ntanios F and Nissen SE: Relationship between cardiovascular risk factors and atherosclerotic disease burden measured by intravascular ultrasound. J Am Coll Cardiol, 2006; 47: 1967-1975

18) Sipahi I, Tuzcu EM, Schoenhagen P, Wolski KE, Nicholls SJ, Balog C, Crowe TD and Nissen SE: Effects of normal, pre-hypertensive, and hypertensive blood pressure levels on progression of coronary atherosclerosis. J Am Coll Cardiol, 2006; 48: 833-838

19) Nicholls SJ, Tuzcu EM, Sipahi I, Schoenhagen P, Hazen SL, Ntanios F, Wun CC and Nissen SE: Effects of obesity on lipid-lowering, anti-inflammatory, and antiatherosclerotic benefits of atorvastatin or pravastatin in patients with coronary artery disease (from the REVERSAL Study). Am J Cardiol, 2006; 97: 1553-1557

20) Thygesen K, Alpert JS, White HD, Jaffe AS, Apple FS, Galvani M, Katus HA, Newby LK, Ravkilde J, Chaitman B, Clemmensen PM, Dellborg M, Hod H, Porela P, Underwood R, Bax JJ, Beller GA, Bonow R, Van der Wall EE, Bassand JP, Wijns W, Ferguson TB, Steg PG, Uretsky BF, Williams DO, Armstrong PW, Antman EM, Fox KA, Hamm CW, Ohman EM, Simoons ML, Poole-Wilson PA, Gurfinkel EP, Lopez-Sendon JL, Pais P, Mendis S, Zhu JR, Wallentin LC, Fernandez-Aviles F, Fox KM, Parkhomenko AN, Priori SG, Tendera M, Voipio-Pulkki LM, Vahanian A, Camm AJ, De Caterina R, Dean V, Dickstein K, Filippatos G, Funck-Brentano C, Hellemans I, Kristensen SD, McGregor K, Sechtem U, Silber S, Tendera M, Widimsky P, Zamorano JL, Morais J, Brener S, Harrington R, Morrow D, Lim M, Martinez-Rios MA, Steinhubl S, Levine GN, Gibler WB, Goff D, Tubaro M, Dudek D and Al-Attar N: Universal definition of myocardial infarction. Circulation, 2007; 116: 2634-2653

21) Mintz GS, Garcia-Garcia HM, Nicholls SJ, Weissman NJ, Bruining N, Crowe T, Tardif JC and Serruys PW: Clinical expert consensus document on standards for acquisition, measurement and reporting of intravascular ultrasound regression/progression studies. EuroIntervention, 2011; 6: 1123-1130, 1129

22) Strandberg TE, Tilvis RS, Pitkala KH and Miettinen TA: Cholesterol and glucose metabolism and recurrent cardiovascular events among the elderly: a prospective study. J Am Coll Cardiol, 2006; 48: 708-714

23) Silbernagel G, Fauler G, Renner W, Landl EM, Hoffmann MM, Winkelmann BR, Boehm BO and Marz W: The relationships of cholesterol metabolism and plasma plant sterols with the severity of coronary artery disease. J Lipid Res, 2009; 50: 334-341

24) Nasu K, Terashima M, Habara M, Ko E, Ito T, Yokota D, Ishizuka S, Kurita T, Kimura M, Kinoshita Y, Asakura Y, Tsuchikane E, Katoh O and Suzuki T: Impact of cholesterol metabolism on coronary plaque vulnerability of tar- 
get vessels: a combined analysis of virtual histology intravascular ultrasound and optical coherence tomography. JACC Cardiovasc Interv, 2013; 6: 746-755

25) Nicholls SJ, Tuzcu EM, Kalidindi S, Wolski K, Moon KW, Sipahi I, Schoenhagen P and Nissen SE: Effect of diabetes on progression of coronary atherosclerosis and arterial remodeling: a pooled analysis of 5 intravascular ultrasound trials. J Am Coll Cardiol, 2008; 52: 255-262

26) Hong YJ, Jeong MH, Choi YH, Ko JS, Lee MG, Kang WY, Lee SE, Kim SH, Park KH, Sim DS, Yoon NS, Yoon HJ, Kim KH, Park HW, Kim JH, Ahn Y, Cho JG, Park JC and Kang JC: Plaque characteristics in culprit lesions and inflammatory status in diabetic acute coronary syndrome patients. JACC Cardiovasc Imaging, 2009; 2: 339349
27) Burke AP, Kolodgie FD, Zieske A, Fowler DR, Weber DK, Varghese PJ, Farb A and Virmani R: Morphologic findings of coronary atherosclerotic plaques in diabetics: a postmortem study. Arterioscler Thromb Vasc Biol, 2004; 24: 1266-1271

28) Moreno PR, Murcia AM, Palacios IF, Leon MN, Bernardi VH, Fuster V and Fallon JT: Coronary composition and macrophage infiltration in atherectomy specimens from patients with diabetes mellitus. Circulation, 2000; 102: 2180-2184

29) Williams KJ, Feig JE and Fisher EA: Rapid regression of atherosclerosis: insights from the clinical and experimental literature. Nat Clin Pract Cardiovasc Med, 2008; 5: 91-102 
Supplemental Table 1. The absolute values of laboratory data at follow-up

\begin{tabular}{|c|c|c|c|c|c|c|}
\hline & \multicolumn{3}{|c|}{ non-DM } & \multicolumn{3}{|c|}{ DM } \\
\hline & Monotherapy $n=36$ & DLLT $n=39$ & $P$ value & Monotherapy $n=13$ & DLLT $n=12$ & $P$ value \\
\hline $\mathrm{TC}, \mathrm{mg} / \mathrm{dL}$ & $141.9 \pm 23.8$ & $129.2 \pm 21.3$ & 0.18 & $139.8 \pm 32.1$ & $121.2 \pm 21.4$ & 0.10 \\
\hline HDL-C, mg/dL & $42.2 \pm 9.5$ & $44.5 \pm 12.8$ & 0.39 & $41.0 \pm 13.5$ & $44.8 \pm 10.0$ & 0.44 \\
\hline LDL-C, mg/dL & $77.6 \pm 17.4$ & $63.7 \pm 16.2$ & 0.001 & $74.2 \pm 25.6$ & $58.3 \pm 16.0$ & 0.08 \\
\hline Triglycerides, mg/dL & $116.0(87.0$ to 144.5$)$ & 97.0 (78.0 to 118.0$)$ & 0.12 & $97.0(84.5$ to 153.0$)$ & $71.5(66.3$ to 96.3$)$ & 0.07 \\
\hline Hs-CRP, mg/L & $0.39(0.21$ to 0.66$)$ & $0.48(0.14$ to 1.63$)$ & 0.78 & 0.35 (0.14 to 0.52$)$ & $0.38(0.22$ to 0.65$)$ & 0.85 \\
\hline Lipoprotein (a), mg/dL & $16.0(7.8$ to 42.3$)$ & $15.0(7.0$ to 36.0$)$ & 0.57 & $10.0(4.0$ to 20.0$)$ & $26.0(8.0$ to 30.8$)$ & 0.94 \\
\hline Apolipoprotein A1, mg/dL & $119.0(106.5$ to 109.0$)$ & $123.0(109.0$ to 134$)$ & 0.29 & $112.0(102.0$ to 131.0$)$ & $119.5(108.5$ to 129.5$)$ & 0.04 \\
\hline Apolipoprotein B, mg/dL & $68.5(58.8$ to 82.3$)$ & $62.0(54.0$ to 70.0$)$ & 0.02 & $74.0(56.0$ to 91.0$)$ & $56.0(49.0$ to 68.3$)$ & 0.44 \\
\hline ApoB/ApoA1 & 0.55 (0.48 to 0.77$)$ & $0.52(0.41$ to 0.59$)$ & 0.05 & $0.63(0.47$ to 0.77$)$ & $0.46(0.42$ to 0.53$)$ & 0.02 \\
\hline Free fatty acid, $\mu \mathrm{Eq} / \mathrm{L}$ & $417.0(282.0$ to 657.0$)$ & $378.0(257.5$ to 551.0$)$ & 0.49 & $380.0(182.0$ to 580.5$)$ & $484.5(338.0$ to 650.5$)$ & 0.27 \\
\hline RLP-C, mg/dL & $3.4(2.7$ to 5.2$)$ & 2.7 (2.1 to 3.5$)$ & 0.22 & $3.3(2.3$ to 4.5$)$ & $2.2(1.7$ to 2.9$)$ & 0.93 \\
\hline sdLDL-C, mg/dL & 20.5 (15.6 to 29.0$)$ & $19.9(13.7$ to 25.9$)$ & 0.19 & $22.9(14.1$ to 31.5$)$ & 15.1 (11.4 to 24.7$)$ & 0.15 \\
\hline Adiponectin, $\mu \mathrm{g} / \mathrm{mL}$ & $5.2(3.3$ to 6.9$)$ & $5.3(3.7$ to 8.0$)$ & 0.70 & $4.2(3.6$ to 5.9$)$ & $5.9(3.8$ to 7.2$)$ & 0.23 \\
\hline HbAlc, $\%$ & $5.6 \pm 0.4$ & $5.4 \pm 0.4$ & 0.15 & $6.3 \pm 0.7$ & $6.5 \pm 0.9$ & 0.54 \\
\hline Lathosterol, mg/dL & 0.7 (0.4 to 1.2$)$ & $0.9(0.7$ to 1.4$)$ & 0.15 & $0.6(0.5$ to 1.0$)$ & $0.9(0.6$ to 1.5$)$ & 0.06 \\
\hline Campesterol, $\mu \mathrm{g} / \mathrm{dL}$ & $5.0(3.3$ to 6.6$)$ & $2.5(2.2$ to 3.3$)$ & $<0.001$ & $4.7(3.5$ to 7.2$)$ & $2.0(1.6$ to 2.4$)$ & $<0.001$ \\
\hline Sitosterol, $\mu \mathrm{g} / \mathrm{dL}$ & 2.5 (1.8 to 3.2$)$ & $1.6(1.2$ to 2.1$)$ & $<0.001$ & $2.3(1.7$ to 3.4$)$ & $1.2(0.9$ to 1.8$)$ & 0.07 \\
\hline Lathosterol, mg/100 mg TC & $0.5(0.3$ to 0.8$)$ & $0.7(0.5$ to 1.1$)$ & 0.03 & $0.5(0.3$ to 0.7$)$ & $0.8(0.6$ to 1.1$)$ & 0.03 \\
\hline Campesterol, mg/100 mg TC & $3.6(2.4$ to 4.3$)$ & 2.1 (1.7 to 2.7$)$ & $<0.001$ & $3.6(2.4$ to 5.6$)$ & $1.6(1.2$ to 2.1$)$ & $<0.001$ \\
\hline Sitosterol, mg/100 mg TC & $1.9(1.3$ to 2.7$)$ & $1.3(1.0$ to 1.6$)$ & $<0.001$ & $1.6(1.2$ to 3.0$)$ & $0.9(0.8$ to 1.6$)$ & 0.05 \\
\hline Campesterol/Lathosterol & $7.3(3.5$ to 14.1$)$ & $2.6(1.9$ to 4.5$)$ & $<0.001$ & $5.6(4.2$ to 18.4$)$ & $1.8(1.3$ to 3.7$)$ & $<0.001$ \\
\hline Plaque volume, $\mathrm{mm}^{3}$ & 81.5 (40.0 to 133.6$)$ & $70.0(36.4$ to 92.1$)$ & 0.16 & $82.6(44.2$ to 120.9$)$ & 87.5 (63.6 to 145.2$)$ & 0.65 \\
\hline $\mathrm{PAV}, \%$ & $51.4 \pm 11.44$ & $48.3 \pm 11.6$ & 0.24 & $51.4 \pm 13.5$ & $55.2 \pm 7.5$ & 0.38 \\
\hline $\mathrm{TAV}, \mathrm{mm}^{3}$ & $92.8 \pm 37.6$ & $81.2 \pm 32.2$ & 0.15 & $109.4 \pm 58.9$ & $105.3 \pm 43.2$ & 0.85 \\
\hline Vessel volume, $\mathrm{mm}^{3}$ & $176.1 \pm 103.5$ & $145.9 \pm 85.8$ & 0.17 & $185.3 \pm 152.3$ & $179.7 \pm 111.5$ & 0.92 \\
\hline Lumen volume, $\mathrm{mm}^{3}$ & $85.6 \pm 52.5$ & $76.3 \pm 49.5$ & 0.43 & $85.4 \pm 65.8$ & $77.7 \pm 47.2$ & 0.74 \\
\hline Lesion length, mm & $12.3 \pm 6.5$ & $10.3 \pm 5.3$ & 0.16 & $10.3 \pm 4.7$ & $11.8 \pm 5.9$ & 0.47 \\
\hline
\end{tabular}

Data are the $n(\%)$, mean $\pm \mathrm{SD}$, or median (IQR). DM, diabetes mellitus; DLLT, dual lipid-lowering therapy with atorvastatin and ezetimibe; MI myocardial infarction; PAD, peripheral artery disease; ACE, angiotensin-converting enzyme; ARB, angiotensin receptor blocker; TC, total cholesterol; HDL-C, high-density lipoprotein-cholesterol; LDL-C, low-density lipoprotein-cholesterol; Hs-CRP, high sensitive C-reactive protein; RLPC, remnant like particle-cholesterol; sdLDL-C, small dense low-density lipoprotein-cholesterol; PAV, percent atheroma volume; TAV, total atheroma volume. 